\title{
Intention to Seek Medical Consultation for Symptoms of Upper Respiratory Tract Infection- A Cross-Sectional Survey
}

\author{
Tan $\mathrm{CE}^{\mathrm{a}}$, Mohd Roozi $\mathrm{AH}^{\mathrm{b}}$, Wong WHR $\mathrm{W}^{\mathrm{b}}$, Sabaruddin $\mathrm{SAH}$, Ghani $\mathrm{NI}^{\mathrm{b}}$, Che Man $\mathrm{Z}^{\mathrm{c}}$ \\ ${ }^{a}$ Department of Family Medicine, Universiti Kebangsaan Malaysia Medical Centre, Jalan Yaacob Latif, 56000 \\ Cheras, Kuala Lumpur. \\ ${ }^{b}$ Medical Students, Department of Family Medicine, Universiti Kebangsaan Malaysia Medical Centre, Jalan \\ Yaacob Latif, 56000 Cheras, Kuala Lumpur. \\ 'Clinical Epidemiology Unit, Universiti Kebangsaan Malaysia Medical Centre, Jalan Yaacob Latif, 56000 \\ Cheras, Kuala Lumpur.
}

\begin{abstract}
Introduction: The common cold is the commonest reason for primary care encounters worldwide. This paper aims to describe the reasons that influence patients to seek medical consultation for the common cold. Methods: This was a cross-sectional survey conducted among adult patients of an urban teaching primary care clinic. An adapted bilingual survey form was administered by the researchers to obtain data regarding their decision to seek medical consultation for a cold and the reasons for their decision. Quantitative analyses were done to describe the close-ended responses. Open-ended responses were analysed using a qualitative approach and the frequencies of the themes were reported. Results: A total of 320 respondents participated in this study, with a response rate of $91.4 \%$. They were predominantly females $(59.4 \%)$, Malay $(70.9 \%)$, and had tertiary education (65.9\%). More than half of the patients $(52.5 \%)$ said they would seek consultation for cold symptoms. Fever was the commonest symptom (57-61\%) which compelled them to seek consultation. The commonest reason for seeking consultation was to get medications $(41.7 \%)$, whereas the commonest reason not to seek consultation was the practice of self-medication (44.2\%). Ethnicity was found to be significantly associated with the decision to seek doctor's consultation. Conclusion: Colds are usually self-limiting and do not result in complications. Empowering patients by providing appropriate self-care knowledge can help to reduce the burden of primary care services. Patients should be taught about red flag symptoms as well as drug safety for medications commonly taken for colds.
\end{abstract}

Keywords: Upper respiratory tract infections, patient acceptance of health care, primary health care.

\section{INTRODUCTION}

Upper respiratory tract infections (URTIs) are the commonest presenting complaint to primary care clinics in Malaysia. ${ }^{1}$ The National Medical Care Survey 2010 reported that URTIs make up nearly a quarter of primary care encounters in Malaysia. The consultation rate for URTIs was 29.6 per 100 consultations. ${ }^{1}$ Worldwide, URTI is the largest contributor to primary care service burden, being the presenting reason for $25-38 \%$ of primary care consultations. ${ }^{2-5}$ The common cold is one of the URTIs.

Corresponding author:

Dr. Tan Chai Eng

MD (UKM), MMed (Family Medicine) UKM

Lecturer

Department of Family Medicine

Universiti Kebangsaan Malaysia Medical Centre

Jalan Yaacob Latif

56000 Cheras, Kuala Lumpur

Tel: +603-9145 $6117(0),+6012-3343145(H P)$

Fax no: +603-9145 9479

E-mail address: tce@ppukm.ukm.edu.my
Previous studies which explored the reasons for seeking medical consultation were done in the United Kingdom as well as Hong Kong. The studies found that patients sought medical consultation because of symptom severity and duration, wish for a diagnosis, reassurance that illness was not severe, peer and family pressure to get treated. ${ }^{6-9}$ Patients also seek consultation if they are worried or lack confidence in self-care, and to obtain sick leave. ${ }^{7}$ Many patients expected to receive antibiotics from their primary care doctor, particularly if their symptoms were severe. ${ }^{6}$

There were also some patients who would not seek medical consultation for a common cold. Reasons given for not seeking medical consultation included mild symptoms which do not interfere with their daily activities and a reluctance to seek help for minor illnesses. ${ }^{6}$ Interestingly, one of the reasons for not seeking medical consultation included knowing that the doctors would not prescribe antibiotics for their condition. ${ }^{6}$

Most colds are self-limiting and resolve with appropriate self-care. ${ }^{9}$ In many developed countries, public awareness towards appropriate self-care for minor ailments have been advocated to reduce the 
burden on primary care service. ${ }^{10}$ Improved public knowledge regarding the nature of common colds and antibiotics led to reduction in number of primary care

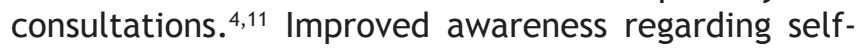
care could allow the primary care services to focus on chronic diseases.

Reasons for seeking consultation in Malaysia may be affected by socio-cultural and health system factors. A local study on help-seeking behaviour among parents when their child had URTI found that gender, ethnicity, socioeconomic status and lack of continuity of care affected the decision to seek consultation for their child when they had a URTI. ${ }^{12}$ However, most studies regarding help-seeking behaviour for URTI revolved around self-medication or antibiotic use. ${ }^{13-16} \mathrm{Few}$ studies explored factors that affect Malaysian patients' decision to seek medical consultation for the common cold or URTI.

This study aims to describe the reasons for patients with symptoms of the common cold to consult a doctor.

\section{METHODOLOGY}

This was a cross-sectional survey conducted at a teaching primary care clinic in Kuala Lumpur Malaysia. All Malaysian patients aged 18 years and above, who came to the clinic for any reason were approached for the study. Patients who had cognitive impairment, hearing and speech difficulties, as well as chronic rhinitis were excluded from this study. Those who could not communicate in English or Malay language

Table I: Survey items were also excluded from the study.

Patients were identified through convenience sampling and were approached at the waiting area for consultation rooms or the clinic pharmacy. Patients were given a brief explanation regarding the study and consent was obtained. From the 350 patients approached, 25 declined and 5 were excluded based on the exclusion criteria. The response rate for this survey was $91.4 \%$. The research team members then administered a prepared bilingual survey questionnaire via face-to-face interview. The survey was coded to protect the anonymity of the respondent.

The survey questionnaire was adapted from a survey on self-care practices done in Canada. ${ }^{17}$ Selected sections of the survey which were relevant to the aims of this study were selected. The original survey form was adapted and translated for this study with permission from the original author. A Malay language version was produced via back-to-back translation and pre-tested among patients attending the hospital's staff clinic to ensure its comprehensibility. This staff clinic catered for all university staff and their immediate family members, including non-healthcare professionals. Revisions were made to the wording of the questionnaire based on results of the pre-test. The adapted bilingual survey form (English and Malay) were used by the research team members to collect information from the respondents. This paper reports part of the results obtained from the study, focusing on patients' intention to seek upper respiratory tract infections (Table I). Respondents were allowed to select more than one response for the second question.

1. When you get a cold, do you see a doctor?

Yes, why:

No, why:

2. If you see a doctor, when do you normally see the doctor for your cold?

As soon as possible

Cough lasts too long

Have coloured phlegm

Fever continues or redevelops

Fever does not respond to fever medication

Persisting sore throat

Difficulty breathing

Chest pain

Others:

Don't know

The quantitative findings of the survey were reported using descriptive statistics such as mean, frequencies and percentages. Open-ended responses were thematically analysed and the frequencies of each theme were reported. Chi square and t-test were used to test the association between the patients' socio-demographic factors and their decision to seek consultation for URTI.
The study received ethical approval from the institutional ethics committee under the Secretariat of Medical Research and Innovation, UKMMC.

\section{RESULTS}

The mean age of the respondents was 47 years. The respondents were predominantly Malay (70.9\%), 
females $(59.4 \%)$ and a majority had at least secondary school education (95\%). Nearly $50 \%$ were unemployed. Table II reports the socio-demographic profile of the respondents and their intention to seek medical consultation for the common cold.

Table II: Socio-demographic profile of the respondents

\begin{tabular}{|c|c|c|}
\hline Sociodemographic factors & \multicolumn{2}{|c|}{$(N=320)$} \\
\hline Mean age + s.d. (years) & \multicolumn{2}{|c|}{$47 . \pm 16$} \\
\hline \multicolumn{3}{|l|}{ Gender,n (\%) } \\
\hline Male & 130 & 40.6 \\
\hline Female & 190 & 59.4 \\
\hline \multicolumn{3}{|l|}{ Ethnicity, n (\%) } \\
\hline Malay & 227 & 70.9 \\
\hline Chinese & 66 & 20.6 \\
\hline Indian & 22 & 6.9 \\
\hline Others & 5 & 1.3 \\
\hline \multicolumn{3}{|l|}{ Education, n (\%) } \\
\hline None & 3 & 0.9 \\
\hline Primary & 13 & 4.1 \\
\hline Secondary & 93 & 29.1 \\
\hline Tertiary & 211 & 65.9 \\
\hline \multicolumn{3}{|l|}{ Occupation, n (\%) } \\
\hline Not working & 162 & 50.6 \\
\hline Employed & 132 & 41.3 \\
\hline Self-employed & 26 & 8.1 \\
\hline
\end{tabular}

More than half of the respondents said they would seek consultation from a doctor if they had cold symptoms. Only $24.1 \%$ said they would not seek consultation from a doctor. About $23.4 \%$ would decide based on the severity or duration of their symptoms. This group was identified through their response to the second question. Although this response was not one of the answers for the first question, we decided to classify this as a separate category for statistical analysis. We believed that these respondents made their decision to consult a doctor based on their situation, and thus did not really belong to the category of those who would or would not seek consultation.

Figure 1 displays the symptoms that prompt patients to seek consultation with a doctor, whereas Figures 2 and 3 display the reasons affecting their decision to seek medical consultation for URTI. The reasons listed in Figures 2 and 3 were derived from the openended responses of the survey. Those who decided to seek consultation based on their symptom severity or duration did not provide additional reasons for seeking consultation.
Most patients said they would see a doctor for their URTI when their fever persisted or recurred (61\%), fever did not subside with antipyretics $(57 \%)$, or when the cough persisted (49\%). The three most common reasons for seeking doctor's consultation were to obtain medications ( $n=70,41.7 \%)$, followed by worries that their condition would worsen $(n=27,16.1 \%)$ and trust in the doctor's treatment $(n=19,11.3 \%)$. On the other hand, the three most frequently reported reasons for not seeing a doctor for their URTI was because they practised self-medication ( $n=34,44.2 \%)$, they felt that the illness was self-limiting $(n=17,14.3 \%)$ and because of long waiting time at the doctor's clinic ( $n=11,14.3 \%)$.

Bivariate analysis showed that ethnicity was significantly associated with the decision to seek doctor's consultation for URTI (see Table III). The proportion of Chinese patients $(39.4 \%)$ who did not intend to seek a doctor's consultation for URTI was significantly more than that of Malays (21.6\%) and Indians (7.4\%). 
Table III: Association between decision to seek doctor's consultation and socio-demographic factors

\begin{tabular}{|lllll|}
\hline Factor & $\begin{array}{l}\text { Yes } \\
(\mathrm{n}=168)\end{array}$ & $\begin{array}{l}\text { No } \\
(\mathrm{n}=77)\end{array}$ & $\begin{array}{l}\text { Depends } \\
(\mathrm{n}=75)\end{array}$ & P value \\
\hline Mean age (years) & 47.8 & 45.7 & 46.9 & $0.619^{\mathrm{a}}$ \\
\hline $\begin{array}{l}\text { Gender } \\
\text { - Male }\end{array}$ & $75(57.7 \%)$ & $29(22.3 \%)$ & $26(20 \%)$ & $0.285^{\mathrm{b}}$ \\
- Female & $93(48.9 \%)$ & $48(25.3 \%)$ & $49(25.8 \%)$ & \\
\hline $\begin{array}{l}\text { Ethnicity } \\
\text { - Malay }\end{array}$ & $119(52.4 \%)$ & $49(21.6 \%)$ & $59(26 \%)$ & $0.005^{\mathrm{b}}$ \\
- Chinese & $30(45.5 \%)$ & $26(39.4 \%)$ & $10(15.2 \%)$ & \\
- Indian \& Others & $19(70.4 \%)$ & $2(7.4 \%)$ & $6(22.2 \%)$ & \\
\hline $\begin{array}{l}\text { Occupation } \\
\text { - Non-employed }\end{array}$ & $83(51.2 \%)$ & $43(26.5 \%)$ & $36(22.2 \%)$ & $0.382^{\mathrm{b}}$ \\
- Employed & $74(56.1 \%)$ & $25(18.9 \%)$ & $33(25 \%)$ & \\
- Self-employed & $11(42.3 \%)$ & $9(34.6 \%)$ & $6(23.1 \%)$ & \\
\hline Education & & & & \\
\hline Primary to secondary & $61(56 \%)$ & $21(19.3 \%)$ & $27(24.8 \%)$ & $0.353^{\mathrm{b}}$ \\
- Tertiary & $107(50.7 \%)$ & $56(26.5 \%)$ & $48(22.7 \%)$ & \\
\hline $\begin{array}{l}\text { a - ANOVA } \\
\text { b - Chi-square test }\end{array}$ & & & & \\
\hline
\end{tabular}

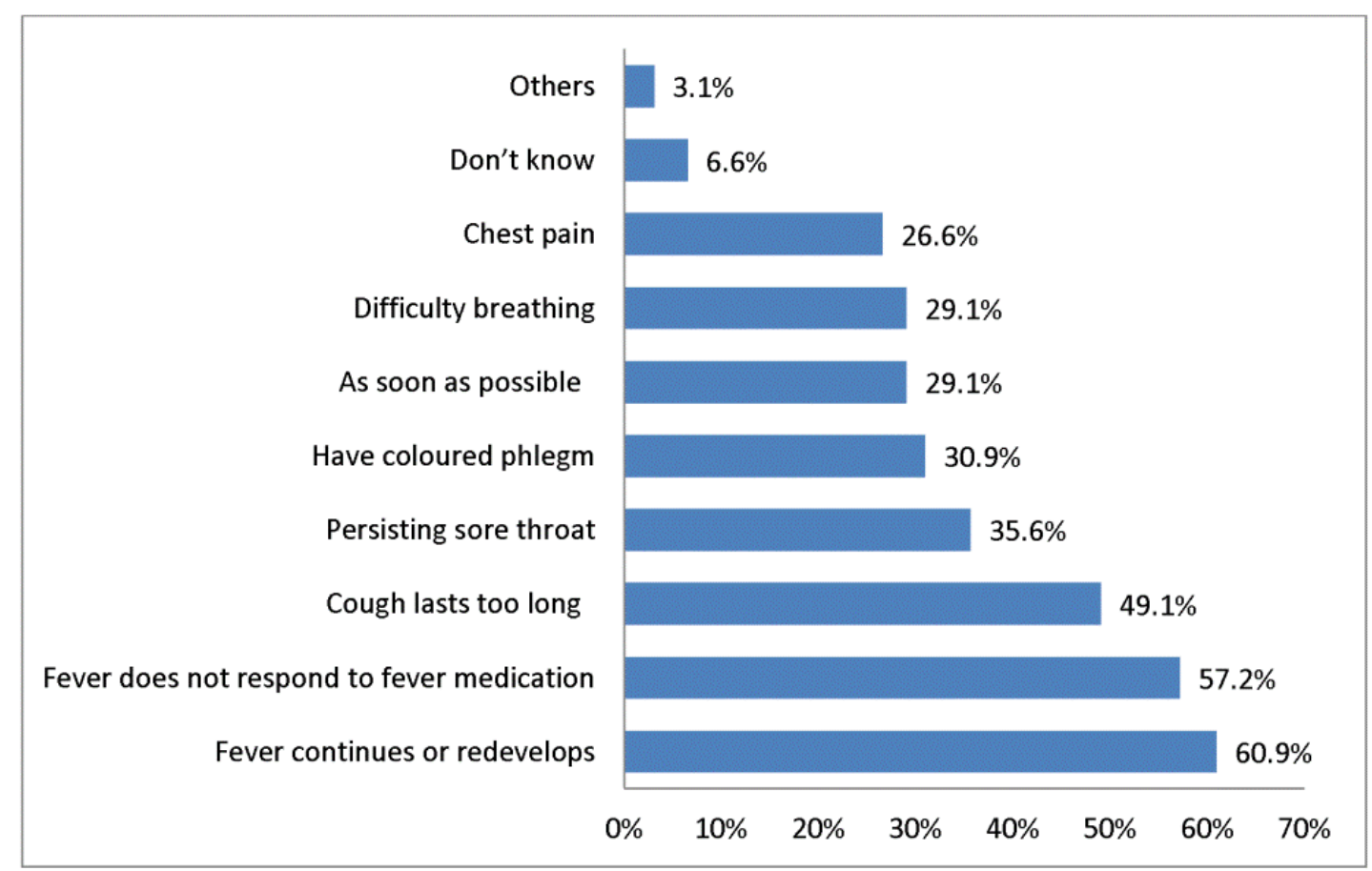

FIGURE 1 : Symptoms that prompt patient to seek consultation 


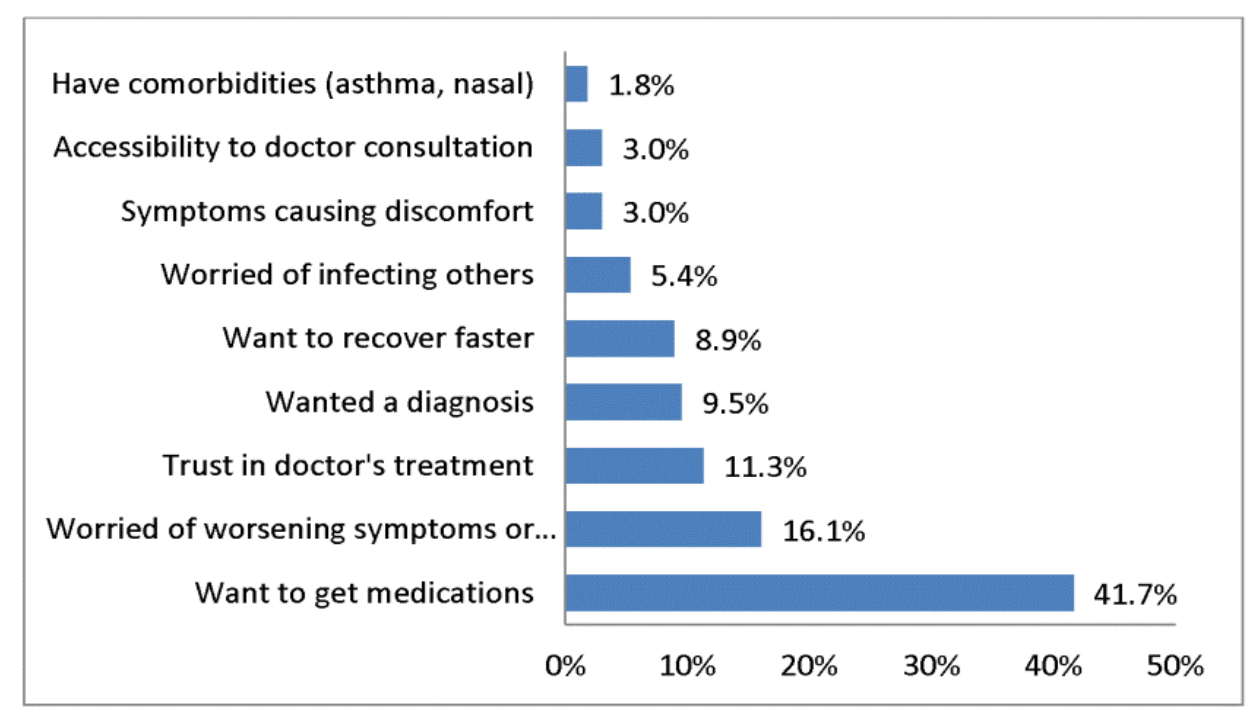

FIGURE 2: Reason for seeking doctor's consultation

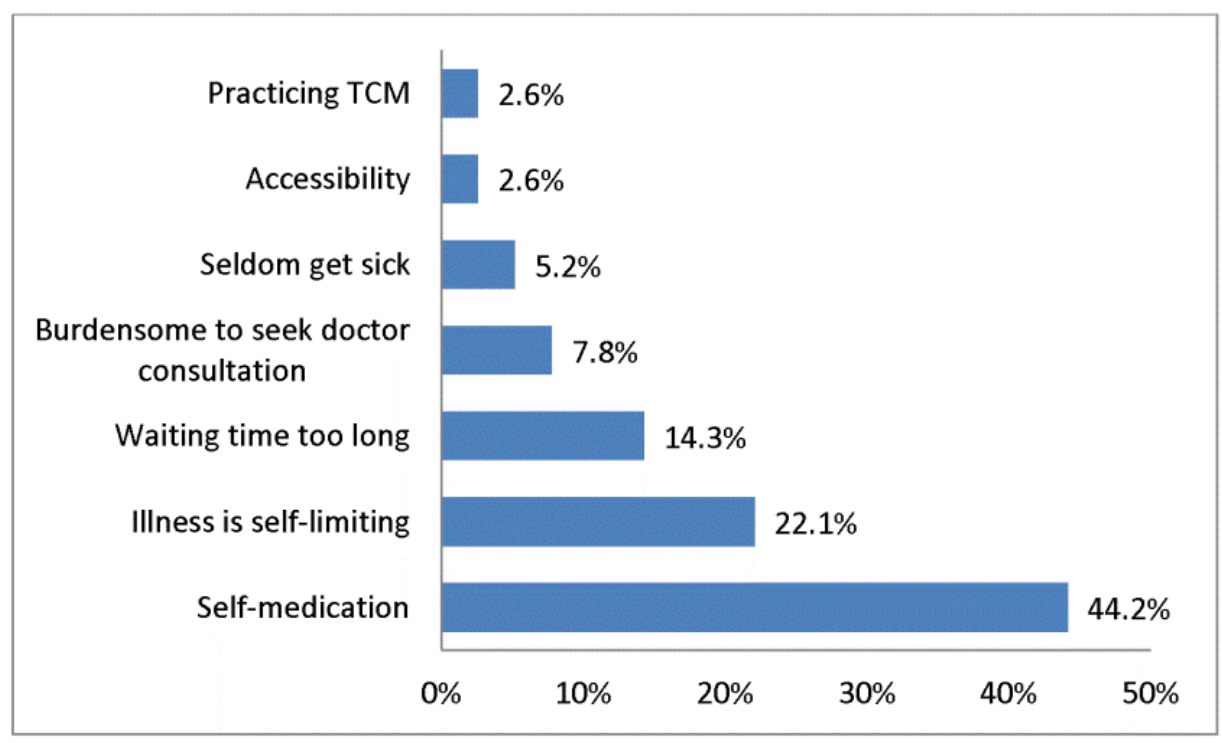

FIGURE 3: Reason for not seeking doctor's consultation

\section{DISCUSSION}

The findings of this study showed that more than half of the respondents felt the need to see a doctor when they developed a cold. Fever was the most common symptom that compelled them to see a doctor. Most patients wanted to see a doctor to obtain treatment for their cold. They believed in the effectiveness of the doctor's treatment for their symptoms. However, ethnicity was found to be significantly affecting their decision to seek a consultation.

Unlike previous studies done in other developed countries, the proportion of respondents who chose to seek doctor's consultation was high.6,18,19 Malaysians preferred to seek consultation for minor ailments such as URTI. ${ }^{12}$ Most of the study participants wanted to see a doctor to obtain medications. Some wanted reassurance that their symptoms were not sinister as they had concerns of developing complications. They had faith in the effectiveness of treatment obtained from the doctor. Patients should be made aware that most colds are self-limiting and seldom result in complications. ${ }^{9}$
In this study, respondents of Chinese ethnicity were less likely to see a doctor for a cold. This concurred with the findings of another local study on parents' help-seeking behaviour when their children had URTI. ${ }^{12}$ Previous studies have shown that the Chinese had more knowledge regarding antibiotics, preferred to self-medicate and had lower rates of healthcare service utilization. ${ }^{12,14,20}$ Further studies to explore the underlying reasons for lower help-seeking behaviour among the Chinese are needed.

This study does not differentiate between appropriate or inappropriate consultations for URTI. The findings found that fever was the commonest symptom that prompted patients to seek medical consultation for URTI. However, red flag symptoms, which may suggest lower respiratory tract infection, were less frequently cited as reasons to seek medical consultation. Conversely, 29\% preferred to see a doctor as soon as possible, despite the fact that majority of URTIs are self-limiting and not complicated. Patient education 
may help patients to recognize red flag symptoms, which are valid causes for concern and prompt them to seek earlier medical consultation. Education about self-care during an episode of URTI also may reduce unnecessary consultations for URTI because of its selflimiting nature. However, further studies are required to see the effectiveness of such interventions. The decreasing trend of doctors consultation in developed countries may result from the effectiveness of public education and efforts to improve doctors' knowledge regarding rational antibiotic usage via local practice guidelines. ${ }^{4,5,21}$ Accessibility to self-care information may help to empower patients to care for themselves without seeking consultation, as well as to improve their awareness of red flag symptoms which necessitates consultation. ${ }^{22,23}$

Self-medication was the most commonly cited reason for not seeking consultation for URTI. Although only a quarter of respondents would not seek consultation for URTI, nearly $45 \%$ said they would self-medicate. About $13 \%$ of Malaysians would self-medicate, whether with over-the-counter medications or prescription medications, without consulting a pharmacist, leading to valid concerns about drug safety. ${ }^{24}$ Knowledge about the dangers of antibiotic use was low among Malaysians, which may lead to problems such as increasing antibiotic resistance and adverse drug reactions. ${ }^{14,15} \mathrm{~A}$ study conducted in urban Indonesia found that only $16 \%$ of patients' self-medication practice was appropriate and $16 \%$ were found to be potentially harmful. ${ }^{25}$ Appropriate self-medication can help to relieve patients' symptoms and reduce healthcare utilisation for minor ailments. However, safety concern regarding the practice of self-medication is a major issue that needs to be considered.

It is also important to note that $29.9 \%$ of patients who chose not to seek consultation cited healthcare service factors (long waiting time 14.3\%, burdensome to seek consultation $7.8 \%$, as well as accessibility $2.6 \%$ ) as their reasons for their decision (see Table III). While it is not recommended that all patients with URTI seek consultation for their symptoms, these results suggest that there is a need to explore regarding the accessibility and patient-friendliness of our healthcare system. These healthcare service factors may be the barrier for timely consultation in potentially serious problems. $^{26}$

There is a need to empower patients to make appropriate decisions regarding the need to seek consultation for cold symptoms. In our efforts to promote self-care among patients for minor ailments such as the common cold, it is also important to ensure that they are well-equipped with knowledge regarding red flag symptoms as well as drug safety. Potential problems which may occur include delayed diagnosis of serious problems, polypharmacy, drug interactions and antibiotic resistance. ${ }^{27}$ Therefore, there is a need for more effective strategies to improve public awareness regarding appropriate self-care measures, when to seek medical consultations, as well as knowledge regarding drug safety and antibiotic use.

This study was conducted among all primary care patients, and not merely those who were having common cold symptoms. It is assumed that almost everyone would have experienced a cold in the preceding 1 year. Therefore, we believe that the results of this study still holds valid as the respondents would make their decisions based on their past experiences. ${ }^{26}$ However, we acknowledge that a community-based study would provide more robust results as we would then be able to include respondents who did not present to primary care for their health problems.

This study has several limitations. Convenience sampling was used which may lead to sampling bias. Furthermore, the patients who came to the primary care clinic may have different help-seeking behaviour compared to those in the community. Therefore, the results may not be truly representative of the general population. A larger nationwide study utilising randomized sampling is needed to confirm the findings of the study. Secondly, the responses given for the open-ended questions were limited, and data saturation was not confirmed. A qualitative study would be more beneficial to fully explore the reasons that affect the patients' decisions to seek consultation for the common cold. Thirdly, the questionnaire used the term 'cold' to refer to symptoms of fever, cough, runny nose, sore throat and others which could represent various types of upper respiratory tract infection. There is a need to check the respondents' understanding regarding the word 'cold' as different interpretations may lead to different responses.

\section{CONCLUSION}

The proportion of patients who seek consultation for URTI in Malaysia is relatively high, compared to developing countries. The commonest reasons for seeking consultation for URTI are to obtain treatment and patient's concerns about worsening symptoms or complications. Effective strategies are needed to empower patients to practice appropriate self-care for URTI and to improve knowledge regarding red flag symptoms which necessitate medical consultation.

\section{ACKNOWLEDGEMENT}

This study received funding from the KMMC Fundamental Research Grant (FF-2014-036). Ethics approval was obtained from the Ethics Committee, Secretariat of Medical Research and Innovations, UKMMC.

\section{Conflicts of interest: None to declare}

\section{REFERENCES}

1. Lim K, Sivasampu S, Goh PP, Hisham A. National Medical Care Statistics 2010. The National Healthcare Statistics Initiative, Clinical Research Centre, Ministry of Health Malaysia. Kuala Lumpur 2012. 
2. Aboulghate A, Abel G, Lyratzopoulos G, Abdelmohsen A, Hamed AR, Roland M. Patterns of disease presentation and management in Egyptian primary care: findings from a survey of 2458 primary care patient consultations. BMC Fam Pract 2013;14:161.

3. Sng QS. Primary Care Survey 2010 - Profile of Primary Care Patients. Ministry of Health, Singapore 2011; 1-4.

4. Gulliford M, Latinovic R, Charlton J, Little P, van Staa T, Ashworth M. Selective decrease in consultations and antibiotic prescribing for acute respiratory tract infections in UK primary care up to 2006. J Public Health 2009; 31:512-20.

5. Neumark T, Brudin L, Engstrom S, Molstad S. Trends in number of consultations and antibiotic prescriptions for respiratory tract infections between 1999 and 2005 in primary healthcare in Kalmar County, Southern Sweden. Scand J Prim Health Care 2009; 27:18-24.

6. McNulty CA, Nichols T, French DP, Joshi P, Butler CC. Expectations for consultations and antibiotics for respiratory tract infection in primary care: the RTI clinical iceberg. Br J Gen Pract 2013; 63:e429-36.

7. Chan CS. What do patients expect from consultations for upper respiratory tract infections? Fam Pract 1996;13:229-35.

8. Linder J, Singer D. Desire for antibiotics and antibiotic prescribing for adults with upper respiratory tract infections. J Gen Intern Med 2003; 18:795-801.

9. Little P, Gould C, Williamson I, Warner G. Reattendance and complications in a randomised trial of prescribing strategies for sore throat: the medicalising effect of prescribing antibiotics. Brit Med J 1997; 315:350-2.

10. Working in Partnership Programme, Proprietary Association of Great Britain. Evaluation of the "Joining Up Self Care" Project - Report to the Working in Partnership Programme. 2006.

11. Ashworth M, Latinovic R, Charlton J, Cox K, Rowlands G, Gulliford M. Why has antibiotic prescribing for respiratory illness declined in primary care? A longitudinal study using the General Practice Research Database. J Public Health 2004; 26:268-74.

12. Ng CJ, Chia YC, Teng CL, Nik-Sherina H. Factors influencing parental decision to consult for children with upper respiratory tract infection. J Paediatr Child Health 2008; 44:208-13.

13. Chan GC, Tang SF. Parental knowledge, attitudes and antibiotic use for acute upper respiratory tract infection in children attending a primary healthcare clinic in Malaysia. Singapore Med J 2006; 47:266-70.

14. Oh AL, Hassali MA, Al-haddad MS, Azhar S, Sulaiman S. Public knowledge and attitudes towards antibiotic usage: a cross-sectional study among the general public in the state of Penang, Malaysia. J Infect Dev Ctries. 2011; 5:338-47.
15. Lim KK, Teh CC. A Cross Sectional Study of Public Knowledge and Attitude towards Antibiotics in Putrajaya, Malaysia. South Med Rev 2012; 5:26-33.

16. Mohamed Azhar MI, Gunasekaran K, Kadirvelu A, Gurtu S, Sadasivan S, Kshatriya BM. Selfmedication: Awareness and Attitude among Malaysian Urban Population. Int J Collab Res Intern Med Public Heal 2013; 5:436-43.

17. Vingilis E, Brown U, Hennen B. Common colds: Reported patters of self-care and health care use. Can Fam Physician 1999; 45:2644-52.

18. Mclsaac WJ, Levine N, Goel V. Visits by adults to family physicians for the common cold. J Fam Pract 1998; 47:366-9.

19. Aoyama I, Koyama S, Hibino H. Self-medication behaviors among Japanese consumers: sex, age, and SES differences and caregivers' attitudes toward their children's health management. Asia Pac Fam Med 2012; 11:7.

20. Krishnaswamy S, Subramaniam K, Low WY, Aziz JA, Indran T, Ramachandran P, et al. Factors contributing to utilization of health care services in Malaysia: a population-based study. Asia Pac J Public Health 2009; 21:442-50.

21. Ashworth $M$, Charlton J, Ballard K, Latinovic $\mathrm{R}$, Gulliford M. Variations in antibiotic rescribing and consultation rates for acute respiratory infection in UK general practices 1995-2000. Br J Gen Pract 2005; 55:603-8.

22. Kemper DW, Lorig K, Mettler M. The effectiveness of medical self-care interventions: a focus on self-initiated responses to symptoms. Patient Educ Couns 1993; 21:29-39.

23. Francis NA, Butler CC, Hood K, Simpson S, Wood $F$, Nuttall J. Effect of using an interactive booklet about childhood. Br Med J 2009; 339: b2885.

24. Hassali MA, Shafie AA, Al-Qazaz H, Tambyappa J, Palaian S, Hariraj V. Self-medication practices among adult population attending community pharmacies in Malaysia: An exploratory study. Int J Clin Pharm 2011; 33: 794-9.

25. Sclafer J, Slamet LS, de Visscher G. Appropriateness of self-medication: method development and testing in urban Indonesia. J Clin Pharm Ther 1997; 22:261-72.

26. Rogers A, Nicolaas $G$. Understanding the Patterns and Processes of Primary Care Use: A Combined Quantitative and Qualitative Approach. Sociol Res Online 1998; 3:5.

27. Hughes CM, McElnay JC, Fleming GF. Benefits and risks of self medication. Drug Safety 2001; 24:1027-37. 
HE INTERNATIONAL MEDICAL JOURNAL MALAYSIA 